This is the author's final, peer-reviewed manuscript as accepted for publication. The publisher-formatted version may be available through the publisher's web site or your institution's library.

\title{
Head-group acylation of monogalactosyldiacylglycerol is a common stress response, and the acyl-galactose acyl composition varies with the plant species and applied stress
}

Hieu Sy Vu, Mary R. Roth, Pamela Tamura, Thilani Samarakoon, Sunitha Shiva, Samuel Honey, Kaleb Lowe, Eric A. Schmelz, Todd D. Williams, and Ruth Welti

\section{How to cite this manuscript}

If you make reference to this version of the manuscript, use the following information:

Vu, H. S., Roth, M. R., Tamura, P., Samarakoon, T., Shiva, S., Honey, S., ... Welti, R. (2014). Head-group acylation of monogalactosyldiacylglycerol is a common stress response, and the acyl-galactose acyl composition varies with the plant species and applied stress. Retrieved from http://krex.ksu.edu

\section{Published Version Information}

Citation: Vu, H. S., Roth, M. R., Tamura, P., Samarakoon, T., Shiva, S., Honey, S., . . . Welti, R. (2014). Head-group acylation of monogalactosyldiacylglycerol is a common stress response, and the acyl-galactose acyl composition varies with the plant species and applied stress. Physiologia Plantarum, 150(4), 517-528.

Copyright: (c) 2013 Scandinavian Plant Physiology Society

Digital Object Identifier (DOI): doi:10.1111/ppl.12132

Publisher's Link: http://onlinelibrary.wiley.com/doi/10.1111/ppl.12132/full

This item was retrieved from the K-State Research Exchange (K-REx), the institutional repository of Kansas State University. K-REx is available at http://krex.ksu.edu 


\section{Head-group acylation of monogalactosyldiacylglycerol is a common stress response, and the acyl-galactose acyl composition varies with the plant species and applied stress}

Hieu Sy Vu ${ }^{1}$, Mary R. Roth ${ }^{1}$, Pamela Tamura ${ }^{1}$, Thilani Samarakoon ${ }^{1,2}$, Sunitha Shiva ${ }^{1}$, Samuel Honey ${ }^{1}$, Kaleb Lowe ${ }^{1}$, Eric A. Schmelz ${ }^{3}$, Todd D. Williams ${ }^{4}$, and Ruth Welti ${ }^{1}$

${ }^{1}$ Kansas Lipidomics Research Center, Division of Biology, Ackert Hall, Kansas State University, Manhattan, KS 66506

${ }^{2}$ Department of Chemistry, Chemistry and Biochemistry Building, Kansas State University, Manhattan, KS 66506

${ }^{3}$ Chemistry Research Unit, Center for Medical, Agricultural, and Veterinary Entomology, US Department of Agriculture-Agricultural Research Service, Gainesville, FL 32608

${ }^{4}$ Mass Spectrometry Laboratory, Malott Hall, University of Kansas, Lawrence, KS 66045

Corresponding author:

Ruth Welti

Division of Biology

Ackert Hall

Kansas State University

Manhattan, KS 66506

$785-532-6241$

Email: welti@ksu.edu 


\begin{abstract}
Formation of galactose-acylated monogalactosyldiacylglycerols has been shown to be induced by leaf homogenization, mechanical wounding, avirulent bacterial infection, and thawing after snap-freezing. Here, lipidomic analysis using mass spectrometry showed that galactose-acylated monogalactosyldiacylglycerols, formed in wheat (Triticum aestivum) and tomato (Solanum lycopersicum) leaves upon wounding, have acyl-galactose profiles that differ from those of wounded Arabidopsis thaliana, indicating that different plant species accumulate different acyl-galactose components in response to the same stress. Additionally, the composition of the acyl-galactose component of Arabidopsis acMGDG depends on the stress treatment. After sub-lethal freezing treatment, acMGDG contained mainly non-oxidized fatty acids esterified to galactose, whereas mostly oxidized fatty acids accumulated on galactose after wounding or bacterial infection. Compositional data are consistent with acMGDG being formed in vivo by transacylation with fatty acids from digalactosyldiacylglycerols. Oxophytodienoic acid, an oxidized fatty acid, was more concentrated on the galactosyl ring of acylated monogalactosyldiacylglycerols than in galactolipids in general. Also, oxidized fatty acid-containing acylated monogalactosyldiacylglycerols increased cumulatively when wounded Arabidopsis leaves were wounded again. These findings suggest that, in Arabidopsis, the pool of galactose-acylated monogalactosyldiacylglycerols may serve to sequester oxidized fatty acids during stress responses.
\end{abstract}

\title{
Abbreviations
}

18:3-20 (and similar abbreviations for fatty acids) Number of carbons: Number of double bond equivalents (excluding the acid carbonyl group) - number of oxygens beyond the carboxylic acid group acMGDG galactose-acylated monogalactosyldiacylglycerol

Arabidopside E 1-OPDA,2-dnOPDA,3-(OPDA-galactosyl) glycerol

Arabidopside G 1-OPDA,2-OPDA,3-(OPDA-galactosyl) glycerol

BHT butylated hydroxytoluene

Col-0 Columbia-0

DAG diacylglycerol

DGDG digalactosyldiacylglycerol 
DGMG digalactosylmonoacylglycerol

ESI electrospray ionization

dnOPDA dinor-oxophytodienoic acid

Gal galactose or galactosyl

JA jasmonic acid

MS mass spectrometry

MGDG monogalactosyldiacylglycerol

MGMG monogalactosylmonoacylglycerol

NL neutral loss

NL 341.2 neutral loss scan of $m / z 341.2$ (and similar abbreviations for neutral loss scans)

OPDA 12-oxophytodienoic acid

PA phosphatidic acid

Pre 277.2 precursor scan of $m / z 277.2$ (and similar abbreviations for precursor scans)

Pst Pseudomonas syringae pv tomato DC3000 expressing AvrRpt2

Q-TOF quadrupole time-of-flight

SQDG sulfoquinovosyldiacylglycerol

\section{Introduction}

Membranes of plant chloroplasts contain glyco-glycerolipids with three major head groups: galactose (Gal, in monogalactosyldiacylglycerol, MGDG), digalactose (in digalactosyldiacylglycerol, DGDG), and sulfonated glucose (in sulfoquinovosyldiacylglycerol, SQDG). The Gal component of MGDG can be enzymatically modified by fatty acylation (esterification) at the 6'-hydroxyl group. Over 40 years ago, this head group acylation was characterized in spinach homogenates (Heinz, 1967a; Heinz \& Tulloch, 1969). Fatty acid compositional analysis of in vitro incubation products from an ammonium sulfate- 
precipitated protein fraction with purified lipid substrates indicated that, when only MGDG was present, galactose-acylated MGDG (acMGDG) was formed via a dismutation reaction, i.e., 2 MGDG $\rightarrow$ acMGDG + monogalactosylmonoacylglycerol (MGMG). However, when both MGDG and DGDG were present, acMGDG was formed exclusively by transacylation from DGDG, i.e., DGDG + MGDG $\rightarrow$ acMGDG + digalactosylmonoacylglycerol (DGMG; Heinz, 1967b; Heinz, 1972). This early work focused on acMGDG formation in homogenized leaf tissues; however, the potential physiological role for the acylation reaction was not considered.

More recently, acMGDGs with the structure 1-(12-oxophytodienoic acid )(OPDA),2-dinoroxophytodienoic acid (dnOPDA),3-(OPDA-Gal) glycerol (Arabidopside E) and acMGDG with 3 OPDA chains (Arabidopside G) were identified in Arabidopsis leaves under stress. These acMGDGs can accumulate to as much as $8 \%$ of the Arabidopsis total leaf lipid when the leaves are infected with the bacteria Pseudomonas syringae carrying the avirulence factor AvrRpt2 (Pst) or AvrRpm1 (Andersson et al., 2006; Kourtchenko et al., 2007). Indeed, in vitro testing indicated that Arabidopsides E and G have antimicrobial activities against the virulent bacterium Pseudomonas syringae DC3000 (Andersson et al., 2006) and the necrotrophic fungus Botrytis cinerea (Kourtchenko et al., 2007). Forty additional acMGDG molecular species (13 non-oxidized and 27 oxidized) were measured after wounding of Arabidopsis leaves (Ibrahim et al., 2011), and 27 additional acMGDGs, each with at least one oxidized fatty acid chain, were characterized as being induced significantly after wounding or avirulent bacterial infection of Arabidopsis leaves (Vu et al., 2012).

Galactolipids with cyclic oxidized acyl chains, or oxylipins, such as OPDA, esterified to glycerol are rare in plant species outside the genus Arabidopsis (Bottcher \& Weiler, 2007). The current study adds to the evidence that, although cyclic fatty acids in membrane lipids may be restricted in occurrence, Gal acylation of MGDG is a relatively conserved process that occurs in tomato and wheat, in addition to Arabidopsis, spinach, and broad bean (Andersson et al., 2006; Heinz, 1967a; Heinz, 1967b; Heinz \& Tulloch, 1969; Heinz, 1972; Ibrahim et al., 2011; Kourtchenko et al., 2007; Vu et al., 2012). MGDG Gal acylation is demonstrated to be a common response to stresses including wounding, freezing, and infection with avirulent bacterial. The data show major variation in composition of the Gal-esterified acyl group, both among plant species and in response to different stresses. Furthermore, comparison of the profiles of the fatty acyl chain on the Gal of acMGDG and the fatty acyl chains of DGDG supports the notion that DGDG is the usual acyl donor for MGDG Gal acylation in vivo. 


\section{Materials and methods}

\section{Plant materials}

Mature wheat leaves (Triticum aestivum L., cultivar Thatcher) were collected from the North Agronomy Farm, Kansas State University, Manhattan, KS. Tomato plants (Solanum lycopersicum, cultivar Better Boy) were purchased from Westside Market, Manhattan, KS. Arabidopsis thaliana accessions Columbia0 (Col-0) and C24 were grown one plant per well in Pro-Mix "PGX" soil (Hummert International, Earth City, MO) in 72-well plug trays (Hummert International, Earth City, MO). Trays were kept in a Conviron growth chamber under a $14 \mathrm{~h} / 10 \mathrm{~h}$ light/dark cycle with $60 \%$ humidity at $21^{\circ} \mathrm{C}$. Light intensity in growth chambers was maintained at $80 \mu \mathrm{mol} \mathrm{m} \mathrm{m}^{-1}$ with cool white fluorescent lights (Sylvania, Danvers, MA). Plants were fertilized twice, once when sowing and once at 20 days old, by irrigation with a $1 \%$ solution of 20-20-20 Miracle-Gro plant food (Scotts Miracle-Gro, Marysville, OH). Col-0 was harvested after 30 days and $\mathrm{C} 24$ after 42 days of growth.

\section{Treatments}

Arabidopsis plants were infected with bacteria (Pseudomonas syringae) as previously described (Vu et al., 2012). Cold acclimation was performed in a $4{ }^{\circ} \mathrm{C}$ room equipped with light carts. Freezing treatment was performed in a programmable freezing chamber (Espec Corporation, Hudsonville, MI). Each tray of plants in soil was partly submerged in an ice slurry (made by adding tap water to $\sim 1.5 \mathrm{~kg}$ of ice chips to a total volume of $4 \mathrm{~L}$ ) to avoid supercooling during freezing treatment at $-8{ }^{\circ} \mathrm{C}$ for $2 \mathrm{~h}$. The soil was completely in contact with the ice slurry through the irrigation holes at the bottom of the growing tray. The temperature was dropped to $-8{ }^{\circ} \mathrm{C}$ without gradual decreasing; at the end of the freezing treatment, plants were transferred to their growth condition $\left(21^{\circ} \mathrm{C}, 60 \%\right.$ humidity) and sampled after $3 \mathrm{~h}$ and $24 \mathrm{~h}$. Leaf numbers 5 and 6 were collected for ion leakage measurement (see next section), and the remaining portion of the rosette was dropped into $4 \mathrm{ml}$ of $75^{\circ} \mathrm{C}$ isopropanol with $0.01 \%$ butylated hydroxytoluene (BHT) for lipid analysis. Leaf number is the order of leaf appearance, determined as described previously (Telfer et al., 1997). Wounding was performed by applying pressure with a hemostat across the leaf midvein, leaving wound marks about $6 \mathrm{~mm}$ apart. For the re-wounding experiment, plants were randomly assigned to one of three groups. Plants of the "control" singly wounded group were harvested at 0 min, 5 min, $15 \mathrm{~min}, 45 \mathrm{~min}, 4 \mathrm{~h}, 24 \mathrm{~h}$ and $48 \mathrm{~h}$ after wounding. For the other two groups, a second wound was applied at the same wounded marks as the first wound either $24 \mathrm{~h}$ or $48 \mathrm{~h}$ after the first wound was applied. The leaves were harvested at $0 \mathrm{~min}, 5 \mathrm{~min}, 15 \mathrm{~min}, 45 \mathrm{~min}, 4 \mathrm{~h}, 24 \mathrm{~h}$ and $48 \mathrm{~h}$ after the second wound. In the re-wounding experiment, four leaves (leaf numbers 5, 6, 7, and 8) were harvested at each 
time point; leaf number 5 was dropped into $2 \mathrm{ml}$ of $75{ }^{\circ} \mathrm{C}$ isopropanol with $0.01 \%$ BHT for lipid analysis, and leaf numbers 6,7 , and 8 were put together into a 1.5-ml tube and frozen in liquid nitrogen for phytohormone analysis by gas chromatography - mass spectrometry (MS).

\section{Ion leakage measurement}

Two leaves from each rosette were rinsed with deionized water before being dropped into a 50-ml glass tube (Pyrex) containing $25 \mathrm{ml}$ of distilled water (Dillons Supermarket, Manhattan, KS). The tubes were shaken for $2 \mathrm{~h}$ at $100 \mathrm{rpm}$ before the first ion conductivity reading with Oakton CON 510 electrical conductivity meter (Oakton Instruments, Vernon Hills, IL). After the first reading, the tubes were incubated at $95-100^{\circ} \mathrm{C}$ in a water bath for $2 \mathrm{~h}$, and a second conductivity reading was taken. Relative ion leakage, as a percentage, was reported as (the first over the second conductivity reading) x 100 (\%).

\section{Lipid extraction}

Modified Bligh-Dyer method (Bligh \& Dyer, 1959) for polar lipid analysis: For the Pst and wounding experiments, three leaves were dropped into $3 \mathrm{ml}$ of $75^{\circ} \mathrm{C}$ isopropanol containing $0.01 \%$ BHT; heating at $75^{\circ} \mathrm{C}$ was continued for $15 \mathrm{~min}$. Chloroform $(1.5 \mathrm{ml})$ and water $(0.6 \mathrm{ml})$ were added, and the tube was shaken for $1 \mathrm{~h}$ before the solvent was transferred to another tube. For the second round of extraction, $4 \mathrm{ml}$ of chloroform: methanol (2:1) were added to the leaves, followed by shaking for $30 \mathrm{~min}$ and combination of the solvent with the previous extract. After repeating the extraction three more times and combining the extracts, the combined extract was evaporated under a nitrogen stream and re-dissolved in $1 \mathrm{ml}$ of chloroform. The extracted leaf residue was dried overnight at $105{ }^{\circ} \mathrm{C}$ and the dry mass obtained by weighing.

Alternate extraction method (for polar lipid analysis): For the freezing and re-wounding experiments, leaves were dropped into a $20-\mathrm{ml}$ vial with a Teflon-lined cap containing $4 \mathrm{ml}$ ( $2 \mathrm{ml}$ in the re-wounding experiment) of $75^{\circ} \mathrm{C}$ isopropanol with $0.01 \%$ BHT. After $15 \mathrm{~min}$ at $75^{\circ} \mathrm{C}, 12 \mathrm{ml}(6 \mathrm{ml}$ in the rewounding experiment) of extraction solvent (chloroform: methanol: $300 \mathrm{mM}$ ammonium acetate in water, 30: $41.5: 3.5, \mathrm{v} / \mathrm{v} / \mathrm{v}$ ) were added, and the tube was shaken at room temperature for $24 \mathrm{~h}$. 
For gas chromatography-MS (free oxylipin analysis): Extraction and derivatization were carried out as described previously (Schmelz et al., 2004).

\section{Mass spectrometry}

For samples extracted by the modified Bligh-Dyer method (stored in $1 \mathrm{ml}$ chloroform), a volume $(x \mu l)$ containing $0.2 \mathrm{mg}$ leaf dry mass was diluted by adding $(360-x) \mu 1$ of chloroform and $840 \mu 1$ of methanol: $300 \mathrm{mM}$ ammonium acetate in water (95: 5, v/v). For samples extracted by the alternate method, a volume $(y \mu l)$ containing $0.2 \mathrm{mg}$ leaf dry mass was diluted with $(1200-y) \mu 1$ of chloroform: methanol: isopropanol: $300 \mathrm{mM}$ ammonium acetate in water (30: 41.5: 25: 3.5, v/v/v/v).

Phospholipids and galactolipids with normal chains were analyzed by triple quadrupole MS using head group-specific scans and standards as described previously (Xiao et al., 2010). Precursor scans of $\mathrm{m} / \mathrm{z}$ 277.2 (Pre 277.2, 18:3), $m / z$ 291.2 (Pre 291.2, 18:4-O), $m / z$ 293.2 (Pre 293.2, 18:3-O), $m / z 295.2$ (Pre 295.2, 18:2-O or 17:3-2O) and $\mathrm{m} / \mathrm{z} 283.2$ (Pre 283.2, 18:0, to detect internal standard 16:0/18:0 MGDG) were performed in negative mode as described previously (Vu et al., 2012), except that $1.505 \mathrm{nmol}$ of 18:0/16:0 MGDG was used in each vial as an internal standard.

Scans for neutral loss (NL) fragments composed of Gal and a fatty acid (Table 1) were carried out in positive mode using an ABI 4000 triple quadrupole mass spectrometer (Applied Biosystems, Foster City, CA) with an electrospray ionization (ESI) source. To perform the NL scans listed in Table 1, three identical sample vials were used to provide enough volume for the analysis of each sample. To each sample vial, $0.95 \mathrm{nmol}$ of di18:0 DGDG was added as an internal standard; this was detected by NL scan of $m / z 341.2$ (NL 341.2), with a target of $m / z$ 966.7. The infusion flow rate was $30 \mu 1 \mathrm{~min}^{-1}$. The scan rate was $36 \mathrm{u} \mathrm{s}^{-1}$ for 75 cycles. Others parameters were: collision gas, 2 (arbitrary units); curtain gas, 20 (arbitrary units); ion source gases 1 and 2, 45 (arbitrary units); source temperature, $100{ }^{\circ} \mathrm{C}$; interface heater, "on"; ion spray voltage, $5500 \mathrm{~V}$; declustering potential, $90 \mathrm{~V}$; entrance potential, $10 \mathrm{~V}$; collision energy, $24 \mathrm{~V}$; and collision cell exit potential, $23 \mathrm{~V}$. 
Accurate acyl mass analysis by quadrupole time-of-flight (Q-TOF) MS was performed on unfractionated lipid extracts with a Q-TOF-2 tandem mass spectrometer (Micromass Ltd., Manchester, UK), using the solvent, parameters, and processing method described by Buseman et al. (2006), with a few changes. Charged precursor ions were subjected to product ion scanning in negative or positive ion mode.

Precursor ions were selected by the quadrupole, tuned to transmit at $0.8 \mathrm{u}$ full width at half height (i.e., monoisotopic selection). Extracts were infused into the ESI source at $20 \mu 1 \mathrm{~min}^{-1}$; collision energy was 30 V.

Chemical ionization gas chromatography - MS was used to profile phytohormones of samples harvested from the re-wounding experiment following the procedure described by Schmelz et al. (2004).

\section{Mass spectral data processing and analysis}

Peak smoothing, background subtraction, and peak centroiding for triple quadrupole MS data were carried out using a custom script with Applied Biosystems Analyst software. After targeted peaks were identified, isotopic overlaps were calculated and subtracted from peaks within each spectrum. For NL scans, spectra were also corrected for isotopic overlaps of head group fragments. Signals of targeted peaks were normalized to the signal of the corresponding internal standard (18:0/16:0 MGDG for negative precursor scans and di18:0 DGDG for positive NL scans) and reported as normalized mass spectral signal per mg of leaf dry mass, where amount of signal produced by 1 nmol internal standard is 1 unit of signal.

To calculate the OPDA to 18:3 signal ratio in MGDGs and DGDGs in Arabidopsis, the ESI triple quadrupole MS signals were detected by scanning in negative mode for Pre 291.2 (OPDA) and Pre 277.2 (18:3). The sum of signals from MGDGs and DGDGs containing combinations of OPDA (18:4-O) with each of the five major fatty acids (16:3, 16:0, dnOPDA (16:4-O), 18:3, and OPDA) was divided by the sum of MGDGs and DGDGs containing combinations of 18:3 with each of the same five major fatty acids. To calculate the Gal-OPDA to Gal-18:3 signal ratio in acMGDGs, the sum of signals of Gal-OPDA acMGDGs (with each of the 35 diacylglycerol (DAG) combinations listed in Table S1), detected by scanning in positive mode for NL 453.3 (Gal-OPDA), was divided by the sum of signals of Gal-18:3 acMGDGs (with the 35 DAGs listed in Table S1), detected by scanning in positive mode for NL 439.3 (Gal-18:3). 
Q-TOF mass spectra obtained in negative mode were mass-corrected by using, as a lock mass, the theoretical exact mass of the acyl anion of 18:3 fatty acid or OPDA, $m / z 277.2173$ or 291.1966, respectively. Q-TOF spectra obtained in positive mode were mass-corrected by locking on the mass of a fragment containing the glycerol backbone attached to either 18:3 fatty acid or OPDA $(\mathrm{m} / \mathrm{z} 335.2581$ or 349.2373, respectively). With the locked mass correction, the exact masses of product ions were determined to ten thousandths of a mass unit.

\section{Results}

\section{Wound-induced acylation of the galactose of MGDG occurs in multiple plant species}

acMGDGs are formed by acylation of MGDG on the carbon at the 6-position of galactose (Heinz \& Tulloch, 1969). Utilizing direct infusion ESI triple quadrupole MS, acMGDG levels can be measured by NL scanning in the positive mode. Fig. 1A depicts an acMGDG molecule, showing formation of the NL fragment, $\mathrm{C}_{22} \mathrm{H}_{43} \mathrm{O}_{6} \mathrm{~N}(417.3 \mathrm{u}$ ), by collision induced dissociation. The fragment is composed of a palmitoyl chain, 16:0 (where 16 is the number of carbons and 0 is the number of double bond equivalents, excluding the carbonyl double bond), esterified to Gal. Other NL fragments used for detection of acMGDGs are listed in Table 1. Each NL scan targets an acMGDG group with a common acyl-Gal component and varied DAG components. The DAG components targeted in each NL scan (Table 1) are listed in Table S1. In contrast to the previous method used by our group to detect acMGDGs by targeting fatty acyl anions (Vu et al., 2012), which did not identify the position of the detected fatty acid among the three positions in acMGDG, the current method detects the fatty acid linked to the galactose. To compare amounts of acMGDGs, signals were normalized to the signal of an internal standard, with an amount of signal equal to that of $1 \mathrm{nmol}$ of the standard equal to 1 . This approach allows sample-to-sample comparison of signals. More detail on the acMGDGs (as defined by DAG species in combination with each acyl-galactose species) may be viewed in Table S2.

Fig. 1B shows that various plant species, from the monocot wheat to eudicots tomato and Arabidopsis, produce acMGDG in response to wounding. acMGDG is formed within 45 min after wounding with a hemostat. Fold increases of acMGDG in leaves 45 min after wounding were 3 for tomato, 18 for wheat, 20 for Arabidopsis C24, and 130 for Arabidopsis Col-0. 
In acMGDG produced in response to wounding, the fatty acyl species linked to Gal varied among plant species (Fig. 2). Fig. 2A shows that in Arabidopsis Col-0, the most abundant Gal-linked fatty acids were 18:4-O, which has been identified as OPDA in galactolipids (Buseman et al., 2006; Stelmach et al., 2001), 16:0, 18:3-2O/20:1, 18:3, and 18:3-O (49\%, 19\%, 11\%, 7\%, and $4 \%$, respectively, of the total acMGDG measured). Gal-linked fatty acids18:3-2O and 20:1 have the same nominal mass and thus are not differentiated by this method. In agreement with previous analyses of Arabidopsis thaliana Col-0, three acMGDGs with the most abundant signals were 1-OPDA,2-dnOPDA,3-(OPDA-galactosyl) glycerol (Arabidopside E, 38\% of total acMGDG signal), 1-OPDA,2-dnOPDA,3-(16:0-galactosyl) glycerol (14\%) and 1,2-diOPDA,3-(OPDA-galactosyl) glycerol (7\%, Arabidopside G) (Table S2; Andersson et al., 2006; Ibrahim et al., 2011; Kourtchenko et al., 2007; Vu et al., 2012). In Arabidopsis C24, Gal linkage of unoxidized fatty acids was more prevalent: 18:3 (32\% of total acMGDG signal), 16:0 (19\%), OPDA (18\%), 18:2 (6\%), 18:3-O (6\%) and 18:3-2O/20:1 (6\%) compared to Col-0 after the same wounding treatment (Fig. 2B). While the amount of acMGDG with OPDA esterified to Gal is $\sim 10$-fold less in Arabidopsis C24 than in Arabidopsis Col-0, the amount of acMGDG with 18:3 esterified to Gal was slightly higher in C24 than in Col-0. Similarly, MS signals from acMGDGs in wounded tomato and wheat leaves were derived primarily from unoxidized Gal-linked fatty acids: 16:0 (40\%), 18:3 (27\%), and 18:2 (15\%) in tomato; 18:3 (73\%), 16:0 (9\%) and 18:2 (6\%) in wheat (Fig. 2C-D). Scanning for NL 439.3 (18:3-containing Gal) in samples from wheat 45 min after wounding produced a massive peak at $\mathrm{m} / \mathrm{z}$ 1052.8, whose signal accounted for $72 \%$ of the total acMGDG signal (Table S2). Accurate-mass product ion analysis of this species (acetate adduct, $\left[\mathrm{M}+\mathrm{C}_{2} \mathrm{H}_{3} \mathrm{O}_{2}\right]^{-}, \mathrm{m} / z$ 1093.8) by Q-TOF MS in the negative mode (Buseman et al., 2006; Vu et al., 2012) showed that this largest acMGDG component of wheat contained only 18:3 acyl chains, consistent with a structure of 1,2-di18:3,3-(18:3-galactosyl) glycerol (Fig. S1). Table S3 shows the acyl composition (three chains) of the major acMGDG molecular species detected in Arabidopsis Col-0, tomato, and wheat and the supporting accurate-mass product ion analysis.

\section{acMGDGs accumulate following stress, including sub-lethal freezing}

The total amounts of acMGDG formed under different stress treatments were determined using the NL scans indicated in Table 1 (Fig. 3). As shown previously by precursor scanning for acyl anions, infection of Arabidopsis thaliana Col-0 with the avirulent bacteria Pst induced large amounts of acMGDG (Fig. 3A; Vu et al., 2012). Wounding also induced acMGDG (Fig. 3B). Similarly, sub-lethal freezing induced synthesis of acMGDG (Fig. 3C). Levels of acMGDG with unoxidized fatty acyl chains had not been 
previously determined (Vu et al., 2012; next section). In the experiment shown in Fig. 3C-E, Arabidopsis Col-0 plants were cold-acclimated at $4{ }^{\circ} \mathrm{C}$ for 3 days or not acclimated (remained at the growth temperature of $\left.21^{\circ} \mathrm{C}\right)$ until the freezing treatment $\left(2 \mathrm{~h}\right.$ at $\left.-8{ }^{\circ} \mathrm{C}\right)$. Plants were returned to $21^{\circ} \mathrm{C}$ after freezing and sampled $3 \mathrm{~h}$ or $24 \mathrm{~h}$ later. As indicated by measurement of ion leakage at $24 \mathrm{~h}$ (Fig. 3D), non-acclimated plants sustained more damage than acclimated plants $(\mathrm{p}<0.001)$. Fig. $3 \mathrm{C}$ indicates that levels of acMGDG increased during the post-freezing period in both acclimated and non-acclimated plants, but that the levels were always higher in non-acclimated than in acclimated plants. Ion leakage measurements (Fig. 3D) indicate that the membranes of acclimated plants were quite permeable at $3 \mathrm{~h}$ into the recovery period, but less so at $24 \mathrm{~h}(\mathrm{p}<0.01)$. In contrast, the non-acclimated plants showed greater leaf membrane damage after $24 \mathrm{~h}$ post freezing than at $3 \mathrm{~h}(\mathrm{p}<0.05)$ and much more damage at $24 \mathrm{~h}$ than observed in acclimated plants. Indeed, acclimated plants sustained visible damage to leaves, but the leaves were able to recover, while the damaged leaves of non-acclimated plants died, although the plant did not (see Fig. S2). Levels of the phospholipid hydrolytic product phosphatidic acid (PA) are shown in Fig. 3E. (Levels of other membrane lipids are shown in Table S4). Whereas total PA levels (Fig. 3E) were closely correlated with leaf ion leakage (Fig. 3D), the total acMGDG signal (Fig. 3C) did not correlate strictly with leaf injury. Acclimated plants tended to accumulate acMGDG between $3 \mathrm{~h}$ and $24 \mathrm{~h}$ $(\mathrm{p}<0.1)$ after freezing treatment as ion leakage dropped. Taken together, comparison of acMGDG signals in acclimated and non-acclimated plants demonstrates a link between treatment and total acMGDG accumulation, but acMGDG accumulated even during recovery.

\section{The composition of induced acMGDGs varies among stresses}

The most abundant acMGDGs in Col-0 leaves after infection of the plants by Pst ( $24 \mathrm{~h})$ were those with Gal-linked fatty acids OPDA (56\%), 16:0 (17\%), 18:3 (10\%), 18:3-2O/20:1 (6\%) and 16:4-O (4\%) (Fig.

4A). The acMGDG composition, with a prevalence of OPDA and 16:0 on Gal, was similar to that formed after wounding (Fig. 4B). The acMGDG Gal-linked acyl composition was drastically different in plants $24 \mathrm{~h}$ after freezing. In acclimated plants (Fig. 4C), 18:3 (48\%), 16:0 (14\%), OPDA (14\%), 16:3 (10\%) and 18:2 (6\%) were most prevalent, and in non-acclimated plants (Fig. 4D), 18:3 (58\%), 16:3 (13\%), 16:0 (11\%), 18:2 (7\%) and OPDA (4\%) were highest. Although acclimated plants accumulated $\sim 5$-fold less acMGDG than non-acclimated plants, they accumulated approximately the same amount of OPDA-Gal acMGDG $\left(0.48 \pm 0.23\right.$ normalized MS units $\mathrm{mg}^{-1}$ dry mass in acclimated plants compared with $0.59 \pm$ 0.17 units in non-acclimated plants). 
Overall, the data demonstrate that during recovery from freezing, as well as during Pst infection and after wounding, significant acylation of the Gal of MGDG was induced. However, in contrast to the composition during other stresses, after freezing, the Gal-linked acyl chains were mostly unoxidized.

\section{The proportions of 16:3, 16:0, and 18:3 in the Gal-esterified acyl chains in acMGDGs resemble proportions in DGDG}

The formation of acMGDG, by a dismutation reaction when MGDG was the only substrate or by transacylation from DGDG when both MGDG and DGDG were present, has been demonstrated in in vitro experiments (Heinz, 1967b; Heinz, 1972). To define the in vivo substrate(s) for acMGDG formation, we considered the acyl compositions of Arabidopsis MGDG and DGDG. Leaf MGDG contains two major molecular species; 18:3/16:3 MGDG is present at higher levels than di18:3 MGDG. Leaf DGDG has three major molecular species: di18:3 DGDG > (16:0/18:3 DGDG + 18:3/16:0 DGDG) > 18:3/16:3 DGDG. The acyl composition of MGDG has previously been analyzed: 59\% 18:3, 33\% 16:3, and only 1\% 16:0, whereas the acyl composition of DGDG contains 77\% 18:3, 3\% 16:3, and 12\% 16:0 (Miquel et al., 1998). Comparing the percentages of 16:0 and 16:3 (and other acyls) esterified to the Gal of acMGDG of stressed (induced) samples to the percentages in MGDG and DGDG of untreated samples should shed light on the origin(s) of the acyl groups (Fig. 5). The fatty acid compositions of MGDG and DGDG used in the current analysis were estimated from the percentage of each MGDG and DGDG molecular species determined by head-group scanning of untreated samples, with assignment of molecular species based on previous product ion analysis (Devaiah et al., 2006). Detailed estimation is shown in Table S5. The percentages of each fatty acid in MGDG and DGDG determined in this way on the untreated samples were in close agreement with the previously published data (Miquel et al., 1998). When acMGDG formation is induced by stresses in Col-0, fatty acid oxidation also occurs at various levels. Hence, for comparison of normal and head group acylated galactolipid compositions, the contents of unoxidized fatty acids and their oxidized derivatives were summed: i.e., 16:3 and its major oxidized derivative, 16:4-O, were combined; similarly, 18:3, 18:4-O, 18:3-O, and 18:3-2O were combined, as were 18:2 and 18:2-2O. The composition of the fatty acids linked to Gal in acMGDG during stress responses, reveals that the percentage of $16: 0$, ranging from $11 \%$ to $19 \%$, is similar to the percentage in DGDG (11\%) and much higher than in MGDG (0.1\%) (Fig. 5). While it is possible that under certain circumstances, some fatty acyl chains used to esterify Gal might come from MGDG, as suggested by the somewhat higher percentage of 16:3 and 16:4-O incorporated on the Gal of acMGDG following freezing stress (Fig. 5E-F), the data are consistent with DGDG as the major source of the Gal-esterified fatty acids in acMGDG formed in vivo. 


\section{The oxidized fatty acyl chain OPDA is enriched on the Gal of acMGDG}

To determine relative amounts of OPDA and 18:3 in MGDG, DGDG, and acMGDG, ratios of MS signals for OPDA and 18:3 in MGDG and DGDG were measured using ESI triple quadrupole MS precursor scanning in negative mode, while ratios of MS signals for OPDA and 18:3 on the Gal of acMGDG were measured using NL scanning in positive mode. Levels of MGDG and DGDG detected by negative precursor scans for 18:4-O (includes OPDA), 18:3-O, and 18:2-O are shown in Table S6. The ratios of OPDA to 18:3 signals under different treatments are shown in Table 2. Pst infection and wounding of Col-0 significantly increased the OPDA level in MGDG and DGDG $(\mathrm{p}<0.001)$ and the OPDA/18:3 signal ratio in MGDG and DGDG $(\mathrm{p}<0.05)$. However, the OPDA/18:3 signal ratio was several orders of magnitude higher on the Gal of acMGDG than in MGDG and DGDG under both induced and noninduced conditions. Although neither acclimated nor non-acclimated plants accumulated much OPDA in galactolipids after freezing treatment, the OPDA/18:3 signal ratio from the acyl chains on the Gal of acMGDG was significantly greater than the OPDA/18:3 signal ratio from the acyl chains esterified to the glycerols of MGDG and DGDG. Interestingly, OPDA enrichment on the Gal of induced acMGDG in cold-acclimated Col-0 plants is greater than in non-acclimated Col-0 recovering from sub-lethal freezing (Table 2). Taken together, the data in Table 2 indicate that the enrichment of OPDA on the Gal of acMGDG is usually correlated with the availability of OPDA in MGDG and DGDG, and OPDA is concentrated in the pool of fatty acids linked to the Gal of acMGDG.

\section{Oxidized acMGDG induction is enhanced by re-wounding}

Although the existence of acMGDG has long been known, its physiological roles are still largely unclear. The fully oxidized acMGDGs Arabidopside E and Arabidopside G have been demonstrated to have antifungal and anti-bacterial activities in vitro (Andersson et al., 2006; Kourtchenko et al., 2007). One hypothesis about acMGDG function is that oxidized-fatty acid-containing complex lipids may serve as reservoirs for precursors of oxylipin-derived phytohormones such as jasmonic acid (JA). In order to test this hypothesis, we wounded Col-0 leaves twice at the same place, with the second wound occurring either $24 \mathrm{~h}$ or $48 \mathrm{~h}$ after the first. Leaves were harvested for lipid extraction at $0 \mathrm{~min}, 5 \mathrm{~min}, 15 \mathrm{~min}, 45$ min, $4 \mathrm{~h}, 24 \mathrm{~h}$ and $48 \mathrm{~h}$ following each wounding event. Harvested leaves were extracted and analyzed for both complex lipids and the free phytohormones JA and OPDA. There was no enhancement by rewounding in levels of induced total free JA and total free OPDA (Fig. S3), indicating that the 
accumulation of pools of esterified oxylipins did not trigger a significantly faster or stronger response in levels of free JA or OPDA upon re-wounding.

Fig. 6 shows levels of plastidic complex lipids MGDG, DGDG, and acMGDG. Whereas free oxylipin content wasn't significantly higher upon re-wounding, signals from oxidized MGDG and DGDG (Fig. 6A-C) were clearly increased by a second wounding to levels higher than by a single wounding. The second wounding did not induce major and sustained increases in signals from unoxidized acMGDG (Fig. 6D-F). In contrast, the levels of oxidized acMGDGs (Fig. 6G-H) were much higher during re-wounding and remained higher than the levels induced by the first wounding for up to $48 \mathrm{~h}$ after re-wounding.

\section{Discussion}

The present work demonstrates that acMGDGs are formed in planta across species (Fig. 1B). Prior work established that wounding and bacterial infection induce acMGDG production in Arabidopsis (Andersson et al., 2006; Kourtchenko et al., 2007; Vu et al., 2012). Homogenization was also reported to induce MGDG acylation in spinach and broad bean leaves (Heinz, 1967a; Heinz, 1972). Here, we demonstrated that acMGDG is also formed in tomato and wheat in response to wounding, suggesting that Gal acylation of MGDG is a conserved response to stress in plants. We also demonstrate that sub-lethal freezing induces acMGDG synthesis in the post-freezing period (Fig. 3C). This was not observed in our previous study, which focused only on oxidized acMGDG and analyzed lipid levels only to the end of the freezing period ( $\mathrm{Vu}$ et al., 2012).

The acyl composition of the acyl-Gal in acMGDG differs in different circumstances. Factors that affect the composition include the plant species, the applied stress, and, likely, other factors that affect the composition of the galactolipid pool. In general the data support the notion that in vivo formation of acMGDG occurs via transacylation from DGDG, as demonstrated previously for in vitro formation (Heinz, 1967b; Heinz, 1972). Species and accessions with more oxidized lipids in the galactolipid pool (Col-0 > C24 > other species) have more oxidized lipids in acMGDG. Stresses that induce more lipid oxidation (bacterial infection and wounding) vs. those that induce less (freezing) also result in production of acMGDG with more oxidized molecular species. Wound-induced acMGDGs containing Gal-linked OPDA (Arabidopsides E and G) were not detected in Brassica napus, Nicotiana tabacum, Pisum sativum, 
Spinacia oleracea, Avena sativa, and barley (Kourtchenko et al., 2007). However, we cannot rule out the possibility that acMGDG is produced in these species with unoxidized fatty acids linked to Gal, similar to the observed reaction products in wheat.

At the same time, the composition of the acyl-Gal in Arabidopsis acMGDG was determined to be more oxidized than the galactolipid acyl pool as a whole. Ibrahim et al. (2011) reported that the ratio of unoxidized acMGDG to oxidized acMGDG in Col-0 leaves harvested $30 \mathrm{~min}$ after wounding (regardless of the positions of oxidized fatty acids on acMGDGs) is 0.6 ; the detection of such a high level of oxidation agrees with our data showing that oxidized fatty acids are enriched in acMGDG. Two possible explanations for the enrichment of OPDA on the Gal of acMGDG are: (1) that an acyltransferase preferentially acylates Gal with an oxidized fatty acid compared to an unoxidized one or (2) that an oxidizing enzyme, such as a lipoxygenase, can act efficiently and directly on an unoxidized fatty acid bound to Gal. The data suggest that, as previously demonstrated in vitro, DGDG in particular is likely to be the source of acyl chains for MGDG acylation to acMGDG in vivo. To date, no protein or gene directly responsible for acylation of acMGDG has been identified. The oxidized MGDGs and DGDGs, such as Arabidopsides A, B, and D (OPDA/dnOPDA MGDG, diOPDA MGDG, and diOPDA DGDG, respectively, Fig. 6A-C), are among the most rapidly formed compounds during stress responses, and the production of acMGDG always lags behind the production of these potential substrate species. This might support the idea of preferential acylation with oxidized fatty acids. On the other hand, Nilsson et al. (2012) presented data suggesting that oxidizing enzymes can directly catalyze oxidation of membrane bound fatty acids. Interaction between a soluble lipoxygenase and a Gal-linked acyl chain might be even more likely. If an oxidizing enzyme could preferentially interact with head group-linked fatty acyl chains, this would support the second possibility.

One potential function for acMGDGs might be as a reservoir for signaling compounds. Another possibility is that acMGDGs are just signs of damage. The current work didn't provide support for either of those possibilities. JA and OPDA production was not directly correlated with acMGDG levels, nor was leaf damage linked with acMGDG levels in the recovery period after freezing. An alternative notion is that the acMGDG pool serves to sequester potentially harmful fatty acids from the main membrane lipid pool. Two examples of accumulation of acMGDG during stress responses support this idea. In the period after freezing, cold-acclimated plants accumulated acMGDG as the leaves recovered and ion leakage decreased (Fig. 3). This acMGDG in acclimated leaves was enriched in oxidized fatty acid more than the 
acMGDG accumulated in non-acclimated leaves which do not recover from freezing damage. The second example is accumulation of acMGDG during re-wounding (Fig. 6). In this case, upon rewounding, levels of acMGDG with oxidized fatty acids linked to Gal appeared to increase more and to stay increased longer than other galactolipid derivatives. These examples imply that acMGDG species are relatively long-lived and may persist and increase as recovery from stress occurs.

\section{Acknowledgements}

The authors would like to thank Dr. Ernst Heinz for his encouragement and helpful comments. We are grateful to Drs. Xuemin Wang and Jyoti Shah for their participation in related studies. We thank Dr. Mark Ungerer for use of a freezing chamber and Dr. Ari Jumponen for use of a light cart. We appreciate Dr. Sunish Sehgal allowing us to harvest wheat leaves from his plantings. This work was supported by the National Science Foundation (MCB 0920663 to R.W.). Instrument acquisition at the Kansas Lipidomics Research Center was supported by National Science Foundation (EPS 0236913, DBI 0521587, DBI 1228622), Kansas Technology Enterprise Corporation, K-IDeA Networks of Biomedical Research Excellence (INBRE) of National Institutes of Health (P20RR16475), and Kansas State University. Contribution no. 13-362-J from the Kansas Agricultural Experiment Station. 


\section{References}

Andersson MX, Hamberg M, Kourtchenko O, Brunnström A, McPhail KL, Gerwick WH, Gobel C, Feussner I, Ellerström M (2006) Oxylipin profiling of the hypersensitive response in Arabidopsis thaliana. Formation of a novel oxo-phytodienoic acid-containing galactolipid, arabidopside E. $J$ Biol Chem 281: 31528-31537

Bligh EG, Dyer WJ (1959) A rapid method for total lipid extraction and purification. Can J Biochem Physiol 37: 911-917

Bottcher C, Weiler EW (2007) cyclo-Oxylipin-galactolipids in plants: occurrence and dynamics. Planta 226: $629-637$

Buseman CM, Tamura P, Sparks AA, Baughman EJ, Maatta S, Zhao J, Roth MR, Esch SW, Shah J, Williams TD, Welti R (2006) Wounding stimulates the accumulation of glycerolipids containing oxophytodienoic acid and dinor-oxophytodienoic acid in Arabidopsis leaves. Plant Physiol 142: 2839

Devaiah SP, Roth MR, Baughman E, Li M, Tamura P, Jeannotte R, Welti R, Wang X (2006) Quantitative profiling of polar glycerolipid species from organs of wild-type Arabidopsis and a PHOSPHOLIPASE D 1 l knockout mutant. Phytochemistry 67: 1907-1924

Heinz E (1967a) Acylgalactosyldiglyceride from leaf homogenates. Biochim Biophys Acta 144: 321-332

Heinz E (1967b) On the enzymatic formation of acylgalactosyldiglyceride. Biochim Biophys Acta 144: 333-343

Heinz E (1972) Some properties of the acyl galactosyl diglyceride-forming enzyme from leaves. Zeitschrift fur Pflanzenphysiologie 69: 359-376

Heinz E, and Tulloch AP (1969) Reinvestigation of the structure of acyl galactosyl diglyceride from spinach leaves. Hoppe-Seyler Zeitschrift fur Physiologische Chemie 350: 493-498

Ibrahim A, Schutz AL, Galano JM, Herrfurth C, Feussner K, Durand T, Brodhun F, Feussner I (2011) The Alphabet of Galactolipids in Arabidopsis thaliana. Front Plant Sci 2: 95 
Kourtchenko O, Andersson MX, Hamberg M, Brunnström A, Gobel C, McPhail KL, Gerwick WH, Feussner I, Ellerström M (2007) Oxo-phytodienoic acid-containing galactolipids in Arabidopsis: jasmonate signaling dependence. Plant Physiol 145: 1658-1669

Miquel M, Cassagne C, Browse J (1998) A new class of Arabidopsis mutants with reduced hexadecatrienoic acid fatty acid levels. Plant Physiol 117: 923-930

Schmelz EA, Engelberth J, Tumlinson JH, Block A, Alborn HT (2004) The use of vapor phase extraction in metabolic profiling of phytohormones and other metabolites. Plant J 39: 790-808

Stelmach BA, Muller A, Hennig P, Gebhardt S, Schubert-Zsilavecz M, Weiler EW (2001) A novel class of oxylipins, sn1-O-(12-oxophytodienoyl)-sn2-O-(hexadecatrienoyl)-monogalactosyl Diglyceride, from Arabidopsis thaliana. J Biol Chem 276: 12832-12838

Telfer A, Bollman KM, Poethig RS (1997) Phase change and the regulation of trichome distribution in Arabidopsis thaliana. Development 124: 645-654

Vu HS, Tamura P, Galeva NA, Chaturvedi R, Roth MR, Williams TD, Wang X, Shah J, Welti R (2012) Direct infusion mass spectrometry of oxylipin-containing Arabidopsis membrane lipids reveals varied patterns in different stress responses. Plant Physiol 158: 324-339

Xiao S, Gao W, Chen QF, Chan SW, Zheng SX, Ma J, Wang M, Welti R, Chye ML (2010) Overexpression of Arabidopsis acyl-CoA binding protein ACBP3 promotes starvation-induced and age-dependent leaf senescence. Plant Cell 22: 1463-1482 


\section{Figures}

Figure 1. acMGDG structure and occurrence upon wounding. A) Structure of a representative acMGDG molecule, 1-18:4-O,2-16:4-O,3-(16:0-galactosyl)glycerol and the fragmentation that gives rise to the NL fragment by collision induced dissociation. A proton moves from right to left during fragmentation. B) Total acMGDG induced by wounding, measured by the 11 NL scans indicated in Table 1, in Arabidopsis Col-0 and C24, tomato, and wheat leaves. Units are in relation to amount of signal detected for $1 \mathrm{nmol}$ of internal standard (di18:0 DGDG), which is denoted as 1. Error bars are standard deviation, $n=5$. The numbers above the bars of wounded samples show the fold induction compared to corresponding unwounded samples.

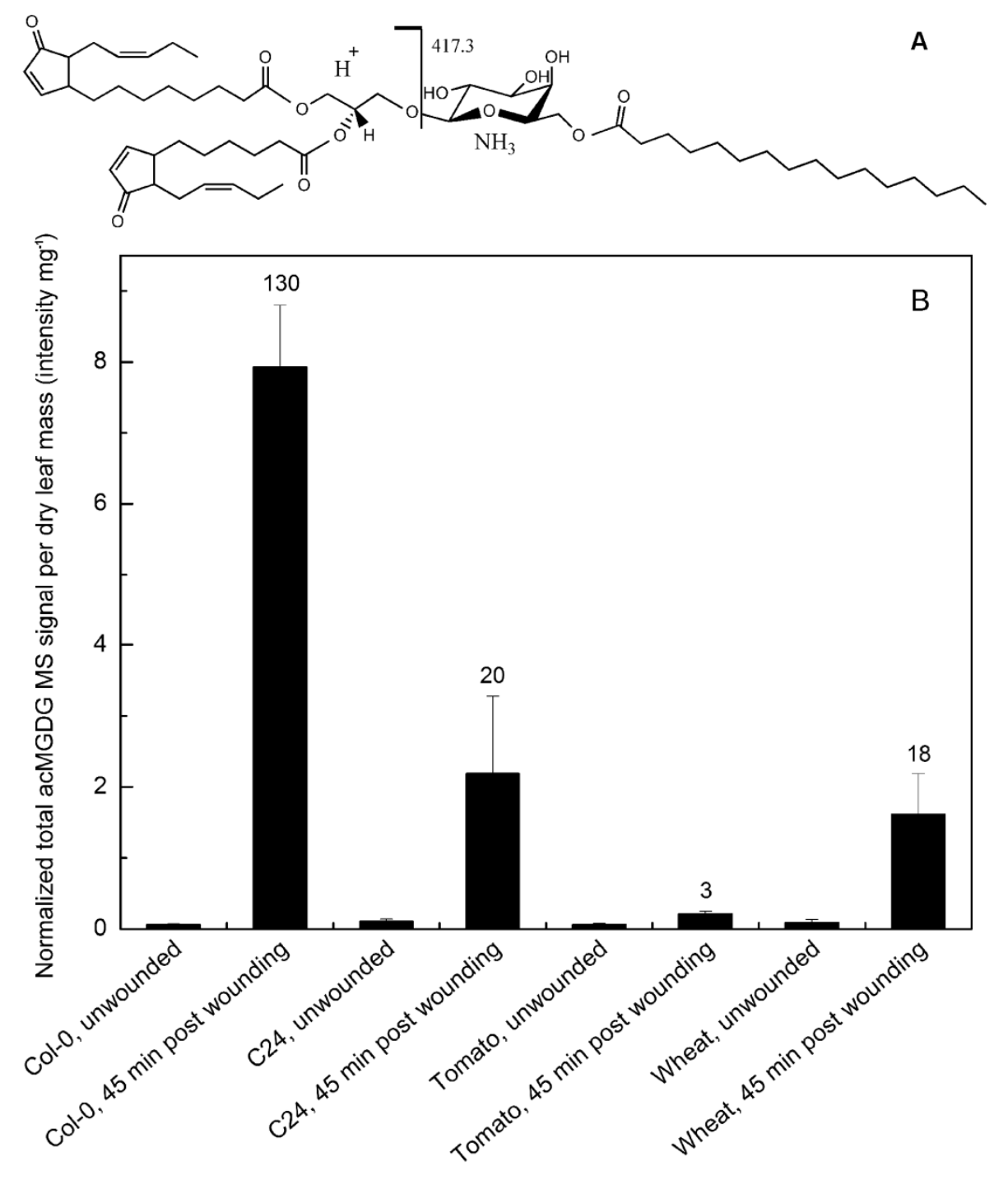


Figure 2. Levels of acMGDG (grouped by fatty acyl moiety on the Gal) in leaves of Arabidopsis Col-0 (A) and C24 (B), tomato (C), and wheat (D) 45 min after wounding. The y axes have different scales. Error bars are standard deviation, $n=5$. The numbers above the bars show the percentage of the corresponding acMGDG group over the sum of the 11 measured acMGDG groups.

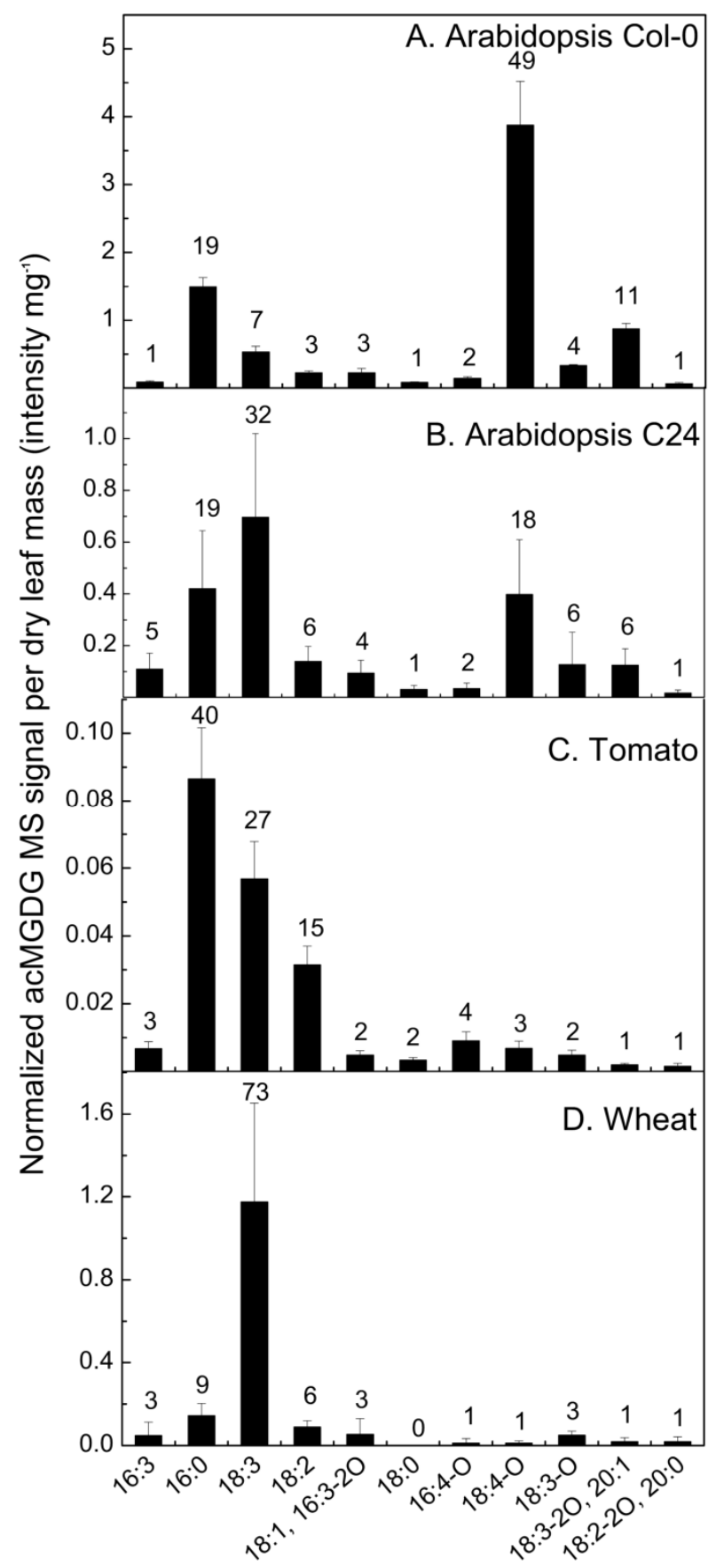

Acyl species linked to Gal 
Figure 3. acMGDG forms in leaves of Arabidopsis Col-0 after application of different stresses, and its occurrence during freezing is not directly associated with cell membrane ion leakage. Panels A, B, and C: acMGDG induced by Pst infection, wounding, and freezing. The y axes have different scales. Panel C shares an x-axis with Panels D and E. Panel D: Relative ion leakage (\%) of acclimated and nonacclimated Arabidopsis Col-0 leaves at $3 \mathrm{~h}$ and $24 \mathrm{~h}$ after freezing treatment. Panel E: Level of total phosphatidic acid as measured by MS in acclimated and non-acclimated Arabidopsis Col-0 leaves at $3 \mathrm{~h}$ and $24 \mathrm{~h}$ after freezing treatment. Error bars are standard deviation; panels A and B: $n=5$; panels C, D, and $\mathrm{E}: \mathrm{n}=6$.

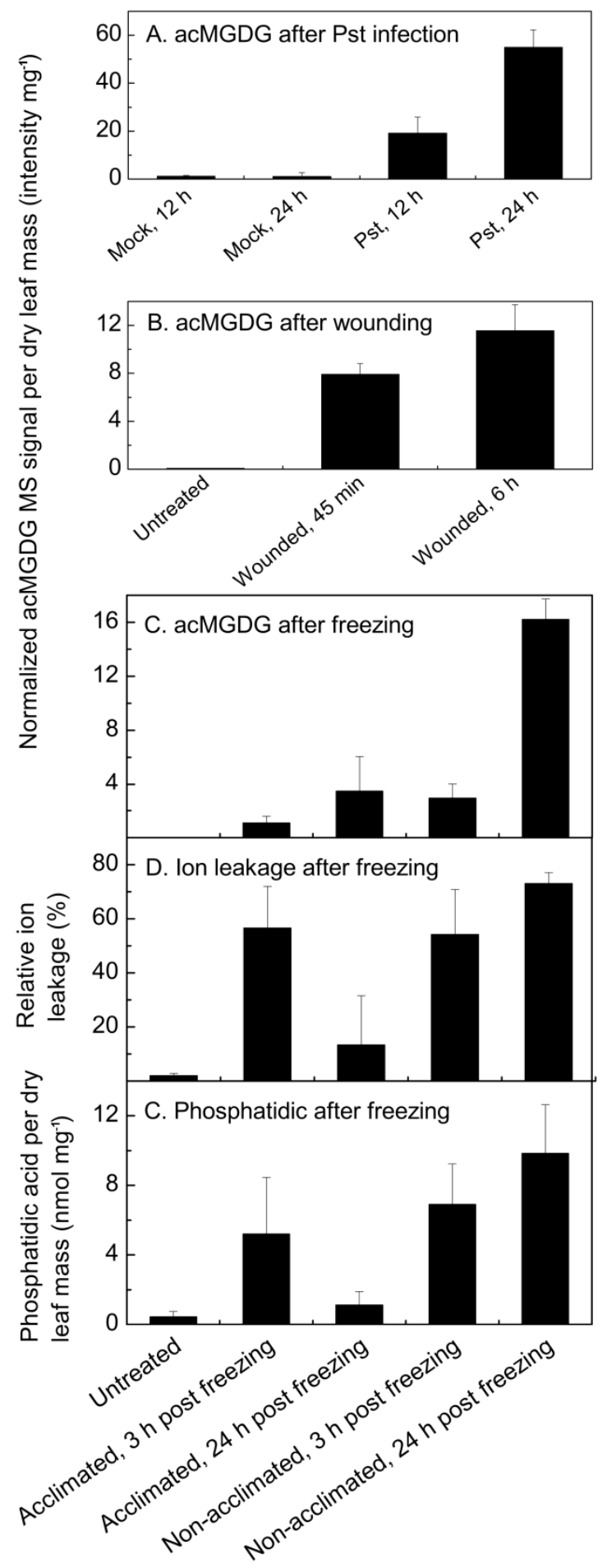


Figure 4. Levels of acMGDG (grouped by fatty acyl moiety on the Gal) in leaves of Arabidopsis Col-0 after application of different stresses. Panel A: acMGDG at $24 \mathrm{~h}$ post Pst infection, $\mathrm{n}=5$; panel B:

acMGDG at 45 min post wounding, $\mathrm{n}=5$; panel $\mathrm{C}$ : acMGDG of cold acclimated Col-0 plants at $24 \mathrm{~h}$ post freezing, $n=6$; panel D: acMGDG of non-acclimated Col-0 plants at $24 \mathrm{~h}$ post freezing, $\mathrm{n}=6$. Y axes have different scales. Error bars are standard deviation. The numbers above the bars show the percentage of the corresponding acMGDG group over the sum of the 11 measured acMGDG groups.

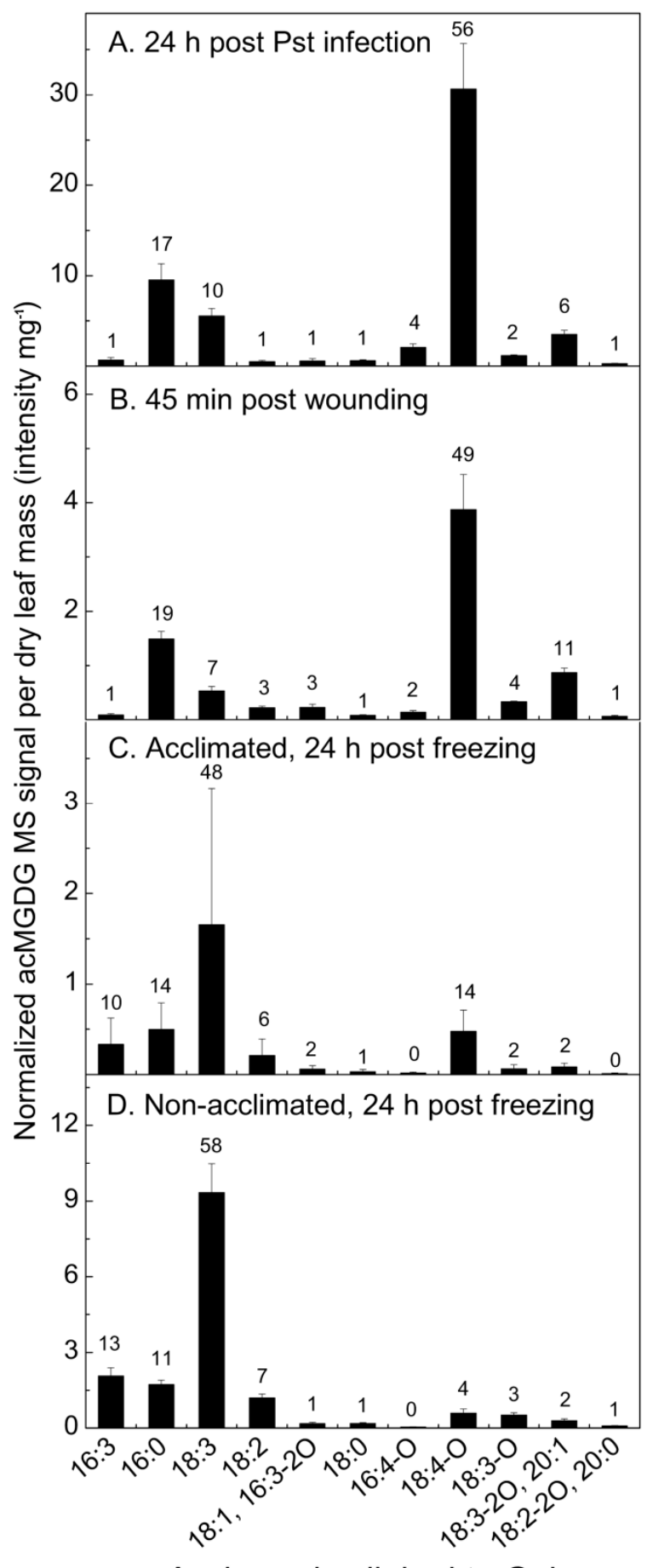

Acyl species linked to Gal 
Figure 5. Fatty acyl composition (\%) of MGDG (A) and DGDG (B) in untreated leaves and fatty acid composition of acyl-Gal in acMGDG of Col-0 plants at $24 \mathrm{~h}$ post Pst infection $(\mathrm{C}), \mathrm{n}=5$; and at 45 min post wounding (D), $\mathrm{n}=5$; of cold acclimated (E) and of non-acclimated (F) Col-0 plants at $24 \mathrm{~h}$ post freezing, $\mathrm{n}=6$.
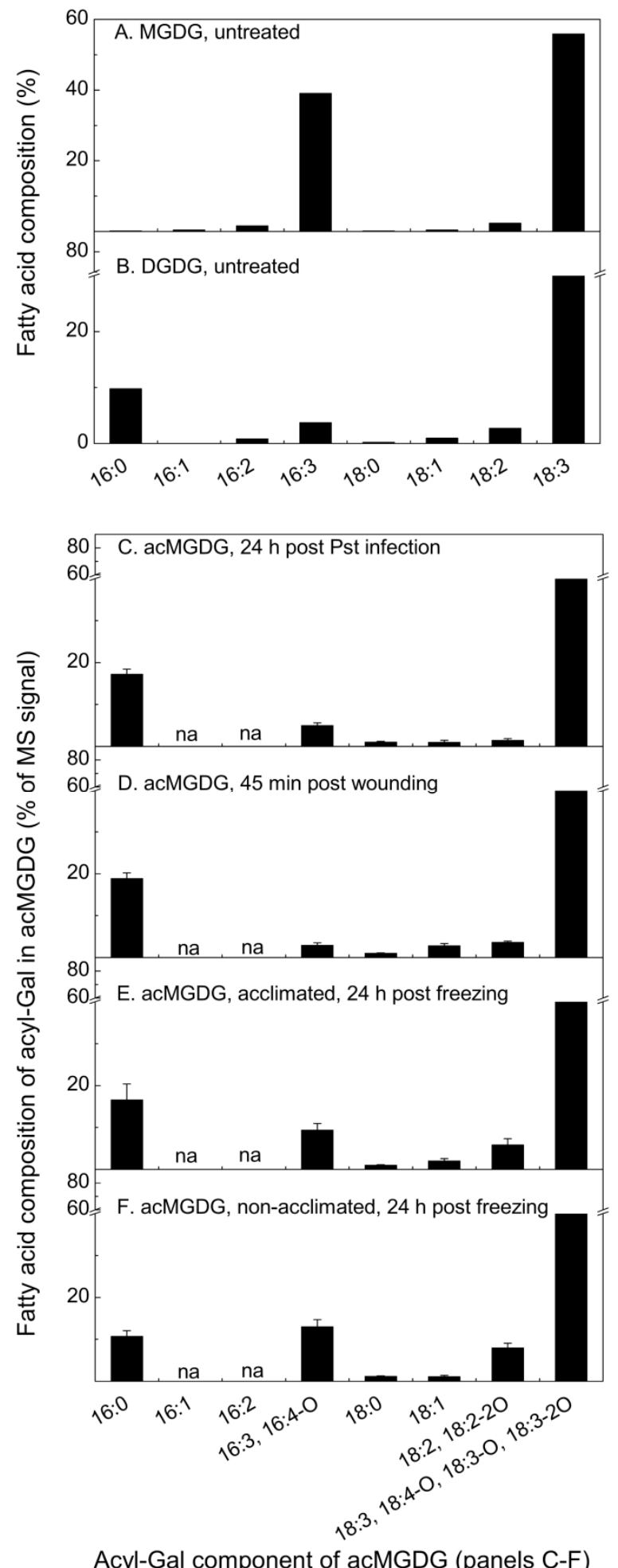
Figure 6. Levels of oxidized MGDG, DGDG, and acMGDG, measured by Pre 277.2 (18:3) and Pre 291.2 (18:4-O) using direct infusion ESI triple quadrupole MS in negative mode. Levels of lipids were measured at various time points after wounding was performed at $0 \mathrm{~h}$ (squares). Data denoted by circles show levels of lipids after a second wounding at the $24 \mathrm{~h}$ time point of the first wounding, and data denoted by triangles show levels of lipids after a second wounding at the $48 \mathrm{~h}$ time point of the first wounding. The $\mathrm{x}$-axis indicates time (h) starting from the only wounding event (squares) or final wounding event (circles and triangles). Y axes are mass spectral signal for the indicated compound, where 1 is the amount of signal detected for $1 \mathrm{nmol}$ of internal standard (18:0/16:0 MGDG), The y axes have different scales. Error bars are standard deviation, $\mathrm{n}=5$.
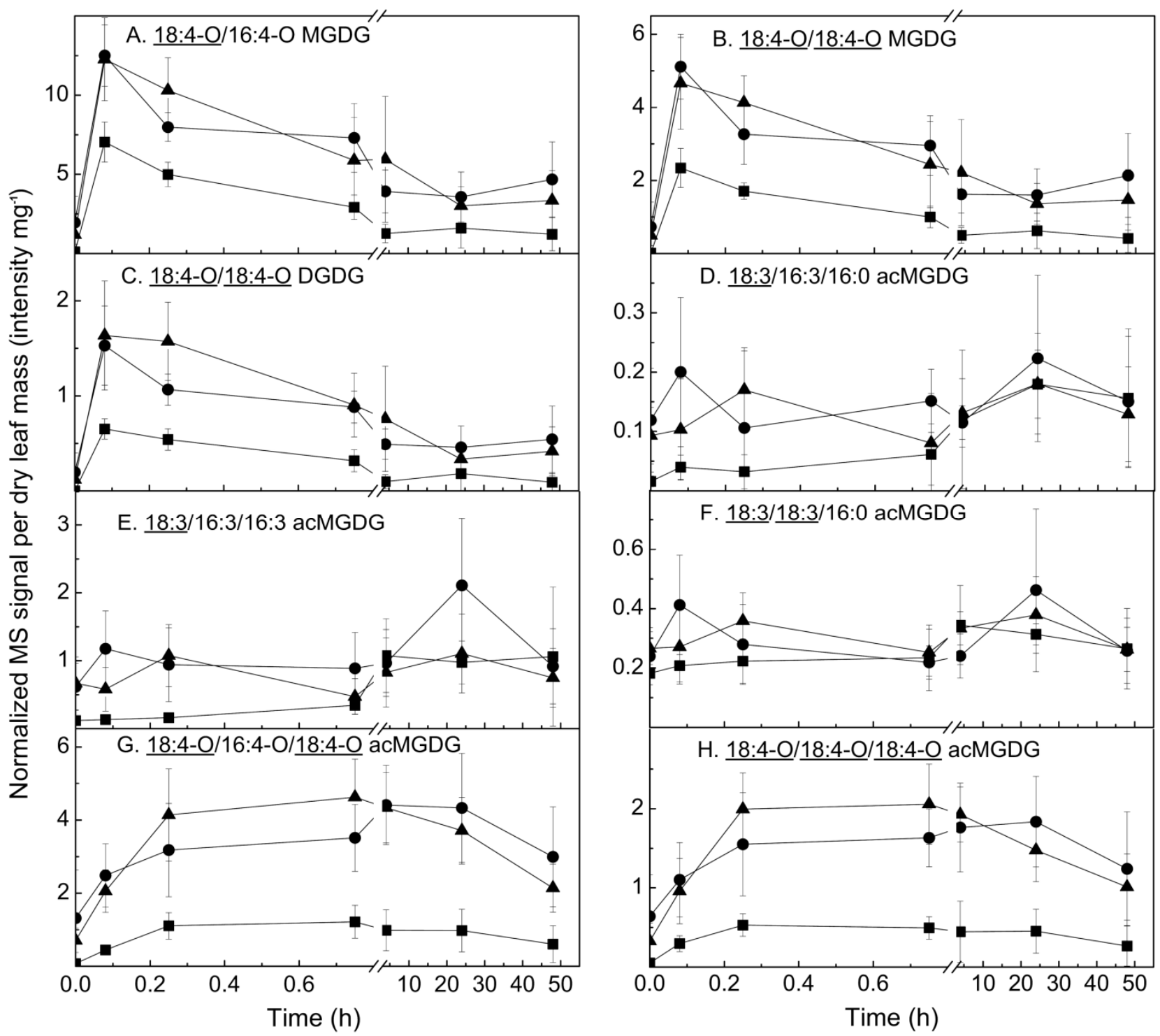


\section{Supporting Information}

Additional Supporting Information may be found in the online version of this article:

\section{“Vu-et-al-2013-Supporting-Information.xls":}

Table S1. DAG fragments of acMGDG determined during NL scanning by ESI triple quadrupole MS in positive mode using scan modes listed in Table 1.

Table S2. Levels of acMGDG detected by NL scans of individual replicates (in normalized mass spectral signal unit $\mathrm{mg}^{-1}$ leaf dry mass).

Table S3. Accurate masses of acyl groups of acMGDG from wounded Col-0, tomato, and wheat provided by Q-TOF MS.

Table S4. Levels of normal chain phospholipids and galactolipids detected by triple quadrupole mass spectrometry (in nmol mg-1 leaf dry mass), performed as described in Xiao et al. (2010, supplemental data).

Table S5. Estimation of fatty acid composition in MGDG and DGDG.

Table S6. Levels of 18:4-O-, 18:3-O- and 18:2-O-containing MGDG, DGDG and phosphatidylglycerol (PG) detected by Pre scans of 291.2, 293.2 and 295.2 in negative mode, as described by Vu et al. (2012).

\section{“Vu-et-al-2013-Supporting-Information.pdf”:}

Figure S1. Tentative structure of 18:3/18:3/18:3 acMGDG detected in wounded wheat leaves.

Figure S2. Acclimated and non-acclimated Arabidopsis thaliana Col-0 after being frozen at $-8{ }^{\circ} \mathrm{C}$ for $2 \mathrm{~h}$.

Figure S3. Total free OPDA and JA after wounding and re-wounding of Col-0 plants. 
Table 1. NL fragments used to detect acMGDGs by ESI triple quadrupole MS in positive mode

\begin{tabular}{lll}
\hline $\boldsymbol{m} / \mathbf{z}$ of NL fragment & Fatty acyl chain & Chemical formula of NL fragment \\
\hline \hline 411.3 & $16: 3$ & $\mathrm{C}_{22} \mathrm{H}_{37} \mathrm{O}_{6} \mathrm{~N}$ \\
417.3 & $16: 0$ & $\mathrm{C}_{22} \mathrm{H}_{43} \mathrm{O}_{6} \mathrm{~N}$ \\
425.3 & $16: 4-\mathrm{O}$ & $\mathrm{C}_{22} \mathrm{H}_{35} \mathrm{O}_{7} \mathrm{~N}$ \\
439.3 & $18: 3$ & $\mathrm{C}_{24} \mathrm{H}_{41} \mathrm{O}_{6} \mathrm{~N}$ \\
441.3 & $18: 2$ & $\mathrm{C}_{24} \mathrm{H}_{43} \mathrm{O}_{6} \mathrm{~N}$ \\
443.3 & $18: 1,16: 3-2 \mathrm{O}$ & $\mathrm{C}_{24} \mathrm{H}_{45} \mathrm{O}_{6} \mathrm{~N}, \mathrm{C}_{22} \mathrm{H}_{37} \mathrm{O}_{7} \mathrm{~N}$ \\
445.3 & $18: 0$ & $\mathrm{C}_{24} \mathrm{H}_{47} \mathrm{O}_{6} \mathrm{~N}$ \\
453.3 & $18: 4-\mathrm{O}$ & $\mathrm{C}_{24} \mathrm{H}_{39} \mathrm{O}_{7} \mathrm{~N}$ \\
455.3 & $18: 3-\mathrm{O}$ & $\mathrm{C}_{24} \mathrm{H}_{41} \mathrm{O}_{7} \mathrm{~N}$ \\
471.3 & $18: 3-2 \mathrm{O}, 20: 1$ & $\mathrm{C}_{24} \mathrm{H}_{41} \mathrm{O}_{8} \mathrm{~N}, \mathrm{C}_{26} \mathrm{H}_{49} \mathrm{O}_{6} \mathrm{~N}$ \\
473.3 & $18: 2-2 \mathrm{O}, 20: 0$ & $\mathrm{C}_{24} \mathrm{H}_{43} \mathrm{O}_{8} \mathrm{~N}, \mathrm{C}_{26} \mathrm{H}_{51} \mathrm{O}_{6} \mathrm{~N}$ \\
\hline
\end{tabular}


Table 2. Ratio of signals from OPDA/18:3 in galactolipids of Arabidopsis thaliana. Total OPDAcontaining MGDG and DGDG (normalized mass spectral signal unit per dry leaf mass), measured by scanning Pre 291.2 in negative mode (complete data in Table S6), are shown in the second column. Col-0 was subjected to Pst infection ("Pst, $24 \mathrm{~h}, \mathrm{n}=5$ ), freezing and post-freezing at $21^{\circ} \mathrm{C}$ with or without prior cold acclimation (“acclimated, 24 h" or "non-acclimated, 24 h”, n = 6), and wounding (“wounded, 45 min”, $n=5$ ). C24 was also wounded and sampled after 45 min ("wounded, 45 min”, $n=5$ ). The third through fifth columns indicate the ratio of signals derived from OPDA to signals derived from 18:3 in acyl chains of MGDG, acyl chains of DGDG, or from the acyl chain on the Gal of acMGDG. Errors are standard deviation.

Treatment

Col-0, untreated

Col-0, Pst, $24 \mathrm{~h}$

Col-0, acclimated, $24 \mathrm{~h}$

Col-0, non-acclimated, $24 \mathrm{~h}$

Col-0, wounded, $45 \mathrm{~min}$

C24, untreated

C24, wounded, 45 min

\begin{tabular}{llll}
\hline OPDA- & \multicolumn{3}{c}{ Ratio of OPDA/18:3 signals } \\
\cline { 2 - 4 } containing & in MGDG & in DGDG & on Gal of \\
MGDG and & & & acMGDG \\
DGDG (intensity & & & \\
mg $^{-1}$ ) & & $0.041 \pm 0.010$ & $5.82 \pm 1.95$ \\
\hline $0.02 \pm 0.01$ & $0.0005 \pm 0.0002$ & $0.0049 \pm 0.0005$ & $5.39 \pm 3.50$ \\
$0.81 \pm 0.15$ & $0.022 \pm 0.006$ & $0.0008 \pm 0.0006$ & $0.51 \pm 0.36$ \\
$0.013 \pm 0.013$ & $0.0006 \pm 0.0010$ & $0.0080 \pm 0.0070$ & $0.063 \pm 0.017$ \\
$0.017 \pm 0.009$ & $0.0092 \pm 0.0080$ & $0.022 \pm 0.011$ & $24.1 \pm 6.5$ \\
$1.26 \pm 0.23$ & $0.0085 \pm 0.0053$ & $0.0027 \pm 0.0026$ & $0.39 \pm 0.21$ \\
$0.17 \pm 0.07$ & $0.0004 \pm 0.0003$ & 0.0027 & \\
$0.26 \pm 0.13$ & $0.0013 \pm 0.0007$ & $0.0058 \pm 0.0018$ & $0.56 \pm 0.07$
\end{tabular}

